\title{
LONG-TERM ACTIVITY IN PHOTOSPHERES OF LOW-MASS STARS WITH STRONG MAGNETIC FIELDS
}

\author{
N. I. Bondar' ${ }^{\prime *}$, M. M. Katsova ${ }^{2 \star}$
}

\begin{abstract}
\section{Keywords}

stars: activity - stars: cycles - stars: starspots - techniques: photometry

1 Crimean Astrophysical Observatory RAS, Nauchny, Russia

2 Sternberg State Astronomical Institute, Lomonosov Moscow State University, Moscow, Russia

e-mail: otbn@mail.ru

**e-mail: maria@sai.msu.ru

Received: March 07, 2020. Accepted: April 29, 2020.
\end{abstract}

The behavior of the average annual luminosity of K-M dwarfs OU Gem, EQ Vir, V1005 Ori and AU Mic was studied at time intervals of several decades. The main sources of photometric data for 1989-2019 were the Hipparcos, ASAS, KWS databases. An analysis of long-term series showed that the average annual brightness of all stars varies cyclical. The durations of possible cycles for OU Gem, V1005 Ori and AU Mic are 40-42 years, for EQ Vir -16.6 years, and amplitudes of cycles are of $0.09-0.2^{m}$. The selected stars belong to the group of red dwarfs for which the average surface magnetic field $\langle B\rangle$ exceeds of several kilogauss. We examined the type of the relationship between the parameters of the cycle, its duration and amplitude, for 9 stars with $\langle B\rangle<4 \mathrm{kG}$. A tendency to increasing of the amplitude and duration of the cycle when the value of $\langle B\rangle$ decrease has been noted. It may be suggested that with an increasing of the surface magnetic field, it becomes more uniform and the level of activity changes in this case in less degrees than on stars with strong local fields concentrated in large spots.

\section{Contents}

1 INTRODUCTION

2 LONG-TERM LIGHT CURVES AND PHOTOMETRIC DATA SOURCES

3 SEARCH FOR POSSIBLE CYCLES IN PHOTOSPHERES OF THE SELECTED STARS

4 ACTIVITY CYCLES AND MAGNETIC FIELDS

5 CONCLUSION

References

\section{INTRODUCTION}

Study the activity of main sequence stars is fundamental importance of both for understanding the development and scales of active phenomena, nature of their generation, as well as some the related to stellar activity issues concerning to studying planets, the origin of life (a habitable zone), large-scale cataclysms, an existence of civilizations.

At present, the instrumental capabilities make it possible to determine the activity indices of $\mathrm{G}-\mathrm{K}$ stars in all layers of the atmosphere and to measure their magnetic fields. Dynamo processes occurring in the convection zone lead to an increase of the magnetic field and its exit to the surface and in upper layers of the atmosphere.

The relationship of parameters of active regions with the physical parameters of stars and rotation has been well studied for solar-type stars with periods of more than 5 days (Baliunas et al., 1995; Oláh et al., 2016; Distefano et al., 2017). On fast-rotating stars with magnetic fields of about $4 \mathrm{kG}$ and more, the activity is less studied, but it has been established that for such strong magnetic fields the relationship between rotation and activity level is disrupt, and an increasing of the rotation does not lead to increase activity indices, i.e., a saturation level is reached (Reiners et al., 2009; Shulyak et al., 2017; 2019). Photometric data for fast-rotating stars were obtained for limited time intervals and for a small set of stars. Currently, in connection with the search for exoplanets, the photometric behavior of $\mathrm{M}$ dwarfs is being actively studied, but in most cases it is data for 7-15 years (Savanov, 2012; Suárez Mascareño et al., 2016; Distefano et al., 2017; Ibañez Bustos et al., 2019). The convective zone of M-dwarfs is deeper than in $\mathrm{G}-\mathrm{K}$ dwarfs, and dwarfs of the spectral class M3.5 and later become fully convective (Chabrier \& Baraffe, 1997). Thus, the conditions for the development of dynamos and magnetic fields in main-sequence stars are different, and to study the activity of stars with different parameters, the accumulation of data on activity indices and magnetic fields remains relevant. 
In this paper, we present the results of the search for cycles at four stars - OU Gem (dK2), EQ Vir (dK5), V1005 Ori $(\mathrm{dK} 0)$, AU Mic (dM1). These are stars with fast rotation and magnetic fields of 2-2.6 kG (Shulyak et al., 2017). For them, the several decade's photometric series were first obtained and studied, and possible activity cycles were revealed. The nature of the relationship between the parameters of the cycle and the average magnetic field for fast-rotating stars (young age) that have not reached saturation is considered by the example of the named group of stars, supplemented by several previously studied objects.

\section{LONG-TERM LIGHT CURVES AND PHOTOMETRIC DATA SOURCES}

The activity cycle associated with the development of sunspots lasts about 11 years. As observations have shown, for solartype stars and smaller masses, longer cycles are possible, and therefore, to search for them it is necessary to study photometric series at intervals of more than 10 years. For the selected stars long-term light curves were formed according to data obtained in photographic archives, published results of photoelectric photometry and taken from the Hipparcos databases, ASAS (Pojmanski, 1997) and KWS (Kamogata/Kiso/Kyoto Wide-field Survey). In the course of statistical processing of arrays of $V$-values selected from the indicated databases, erroneous values and flares were identified and removed, average brightness values for each observation date, average annual values and errors of their determination were determined. The mean square errors of $V_{y r}-$ values did not exceed $0.07^{m}$ according to photographic data, $0.015^{m}$ according to photoelectric data, $0.015^{m}, 0.03^{m}, 0.028^{m}$ according to Hipparcos, ASAS and KWS, respectively.

For the star OU Gem it were used photographic magnitudes for 1899-1988 (Bondar', 1995), electrophotometry collected by Alekseev (2001) obtaining from his observations at the $1.25 \mathrm{~m}$ AZT-11 telescope and taken from publications (1972-1988), and results from databases Hipparcos (19901992), ASAS (2000-2009) and KWS (2010-2019). The $B$ magnitudes added from photographic data were converted to $V$-values according to the corresponded $B-V$-values.

The complete light curve in Fig. 1 covers the time interval of 120 years, from 1899 to 2019 , the number of average annual $V$-values $N=56$. The brightness changes from year to year amounted to $0.2^{m}$; the maximum level (marked by a dashed line) was reached in 1962 and 1964 (Argue, 1963) and 2004 (ASAS).

The behavior of the average annual brightness of EQ Vir is presented on the interval 1989-2019 $(N=25)$ by photometry from the Hipparcos (1989-1993), ASAS (2000-2009) and KWS databases (2010-2019) (Fig. 1). Changes in the brightness amounted of $0.22^{m}$. The photographic data for 19141988 (Bondar', 1995) are not numerous and very sparsed, they were not included in the data array for a search for the cycle, but we took into account, that on this interval and also in 1993-1998 (Alekseev, 2001), the brightness did not reach its maximum of $V=9.20^{m}$ that was found from ASAS and KWS data.

For star V1005 Ori, the results of 1974-1976 obtained by Bopp and Espenak (1977), Bopp et al. (1978), data for 1980-1981 by Byrne et al. (1984) and 1992-1999 (Alekseev, 2001) were taken, and $V$-values from Hipparcos (1990-1993), ASAS (2001-2009) and KWS (2010-2019) as well. The average annual values from the Hipparcos catalog were recalculated, $V_{y r}=V_{H i p}-0.11$ correspondently to the data from (Alekseev, 2001). The light curve in Fig. 1 includes 34 epochs of observations. Changes in the average annual brightness from 1974 to 2019 amounted to $0.19^{m}$, on the entire studied interval the maximum brightness level of $9.25^{m}$ was observed only once, in 1994.

The AU Mic light curve (Fig. 1) covers the interval from 1972 to 2018 . The yearly mean values $(N=32)$ were obtained from data by Cutispoto (1995; 1998), Cutispoto et al. (2001; 2003) Hipparcos (1990-1992), ASAS (2001-2009) and KWS (2013-2018). Changes in the average annual brightness were $0.27^{m}$, and the maximum brightness was $8.59^{m}$ in 2000. According to archival data from Phillips and Hartmann (1978), this level was not reached on the span from 1913 to 1971. In Table 1, we present the maximum magnitudes of stars obtained by us in the studied time interval and the data from the SIMBAD.

\section{SEARCH FOR POSSIBLE CYCLES IN PHOTOSPHERES OF THE SELECTED STARS}

Long-term light curves make it possible to determine the epochs of maximum brightness and the amplitude of variability. Changes in the average annual brightness for active solartype stars and red dwarfs are explained in the frameworks of the concept of the development of surface starspots. The epochs of minimum brightness correspond to the maximum spottedness (maximum activity), and spots on the surface of the star are absent or their number is minimal in the years of the greatest brightness. The interval between successive epochs of the star's maximum brightness (activity minima) shows the duration of the possible cycle of its photospheric activity. The stellar activity cycle can last several decades and, according to available observations, it is rarely possible to trace the development of several cycles. Therefore, the found long-term cycles should now be considered possible or suspected, but their search allows us to identify stars with cycles and irregular activity, which is important for studying the nature of the magnetic activity of stars.

The search for periods in brightness variations for the stars considered by us was performed used the methods of Yurkevich and Hartley as described early by Bondar' (2019). For OU Gem, for a number of average annual $V$-values, the presence of periods of less than 60 years was considered; the best data convolution was obtained for a period of 41.6 years. Figure 3 shows the phase curve for this period and the approximation of the average annual values in bins of 0.1 

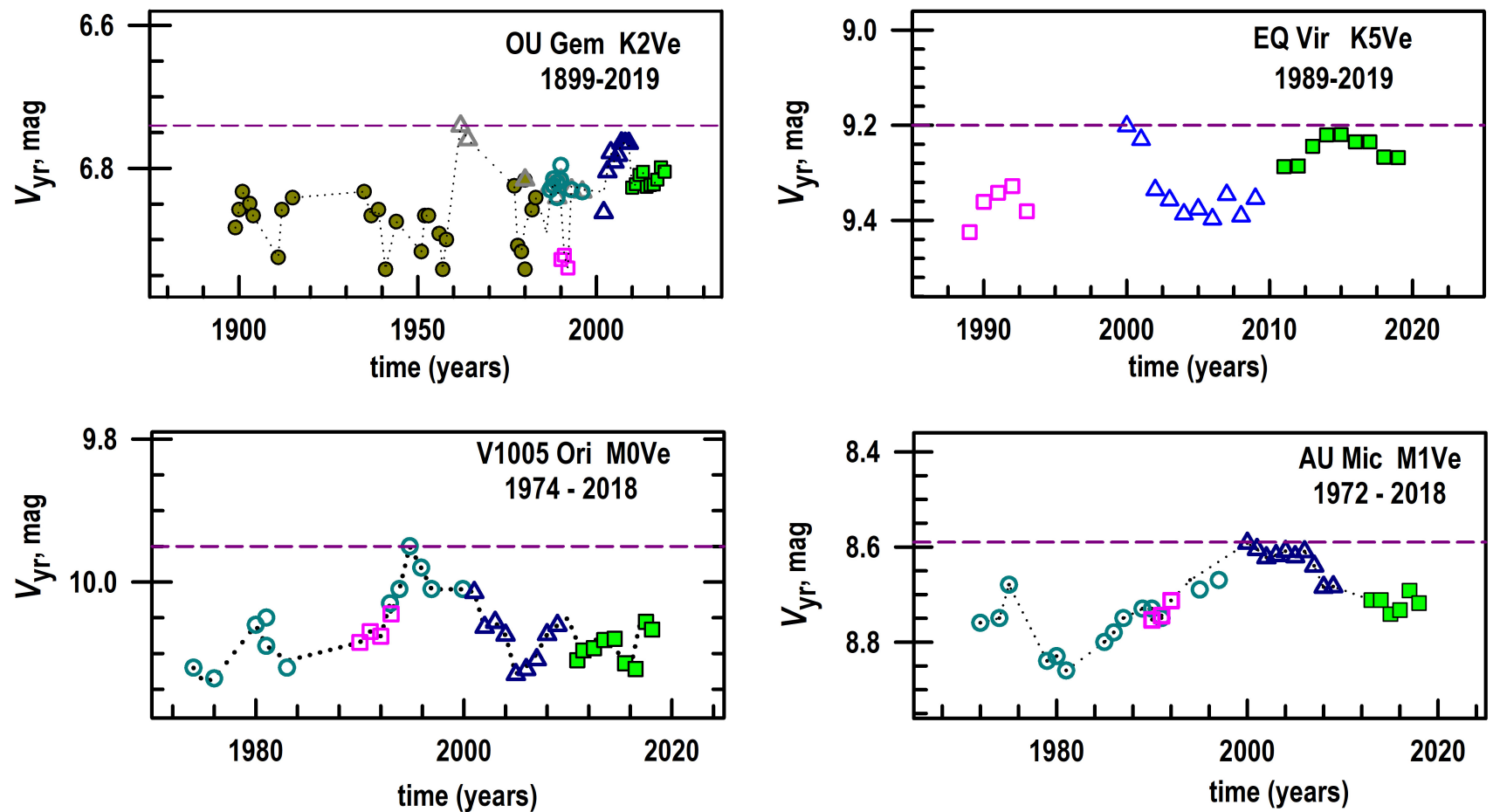

Figure 1. Changes in the average annual brightness at long-term intervals for active red dwarfs OU Gem, EQ Vir, V1005 Ori, AU Mic. Different symbols indicate the following data sources: filled circles - photographic collection of Sternberg Astronomical Institute, Moscow State University (Bondar', 1995), open circles - published photometry data by Cutispoto $(1995,1998)$ and Cutispoto et al. (2001, 2003), open squares - catalog of Hipparcos satellite data, open triangles - ASAS photometric catalog, filled squares - KWS database. The dashed line marks the level of maximum brightness on the presented observational interval.

Table 1. Maximal $V$-magnitudes for investigated stars

\begin{tabular}{|c|c|c|c|c|}
\hline star name & $\begin{array}{c}\text { time } \\
\text { interval }\end{array}$ & $\begin{array}{c}V, \text { mag } \\
(\text { SIMBAD) }\end{array}$ & $V_{\max }$, mag & $\begin{array}{c}\text { years of maximum } \\
\text { in } V_{y r} \text {-values }\end{array}$ \\
\hline OU Gem & $1899-2019$ & - & 6.74 & 1962,2007 \\
\hline EQ Vir & $1989-2019$ & 9.37 & 9.20 & 2000,2015 \\
\hline V1005 Ori & $1974-2018$ & 10.11 & 9.95 & 1994 \\
\hline AU Mic & $1972-2018$ & 8.63 & 8.59 & 2000 \\
\hline
\end{tabular}

phases by a $5^{\text {th }}$ degree polynomial (dashed line), the initial epoch is chosen arbitrarily, $T_{0}=1899$. The amplitude of the possible cycle is $0.09^{m}$.

For EQ Vir, we analyzed a series of averaged $V$-magnitudes for each date of observations (Fig. 2). According to the ASAS and KWS data, the possible cycle $\left(P_{c y c}\right)$ on the interval JD2451900-2458569 was determined as equals to 6095.62 days. The Hipparcos data are corresponded to the convolution with this period, and we have precised the value of the possible cycle for the entire data on the interval JD 2447868 $2458569\left(T_{0}=2447868.49, N=874\right)$. With the obtained $P_{c y c}=6059.53$ days ( 16.59 years), two waves of this cycle were observed. They are shown by the corresponding icons in the left graph of Fig. 2. It can be seen that the shape and phase of the cycle are not changed. A convolution of the mean yearly $V$-values is shown in Fig. 3, $T_{0}=1989$, the approxima- tion by bins equal to 0.1 of the phase represents the shape of the found cycle and its amplitude $A_{c y c}=0.19^{m}$.

For the M-dwarfs V1005 Ori and AU Mic, the search for possible periods is complicated by the fact that only one epoch of maximum brightness was recorded on the studied intervals for these stars. Periodogram analysis was performed for a series of average annual $V$-values and for the row obtained by approximating the light curve with a high degree polynomial. The best convolution of the yearly mean $V$ values was obtained for V1005 Ori with the period of 38.06 years $\left(T_{0}=1974\right)$, for AU Mic - with a period of 40.6 years $\left(T_{0}=1972\right)$ (Fig. 3). The amplitude of the suspected cycles is $0.13^{m}$ and $0.21^{m}$, respectively. Approximation by polynomials has been carried out according to the average values in bins equal to 0.1 of the phase of the accepted periods. The parameters of the discussed activity cycles are shown in Table 2 . 

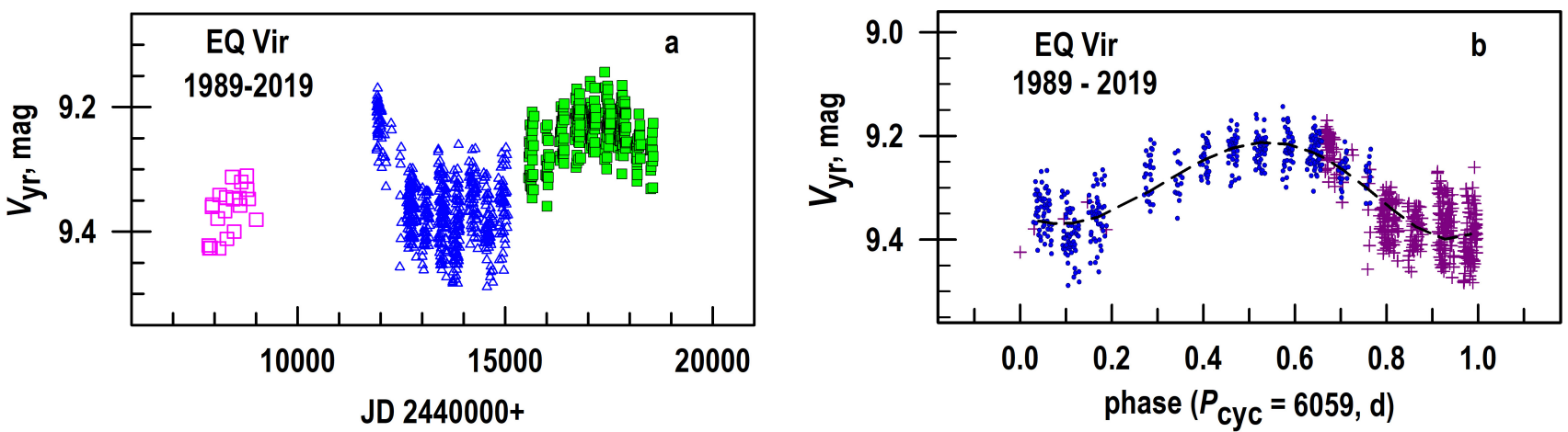

Figure 2. Activity cycle in photosphere of EQ Vir from $V$-data obtained in 1989-2019. a) Average $V$-values for the date of observations according to the data from Hipparcos, ASAS and KWS, description to symbols as in Fig. 1. b) The convolution of the averaged $V$-data with $T_{0}(J D)=2447868$ and the found cycle of $6059.53 \mathrm{~d}$ shows two consecutive cycles marked with dots and crosses.
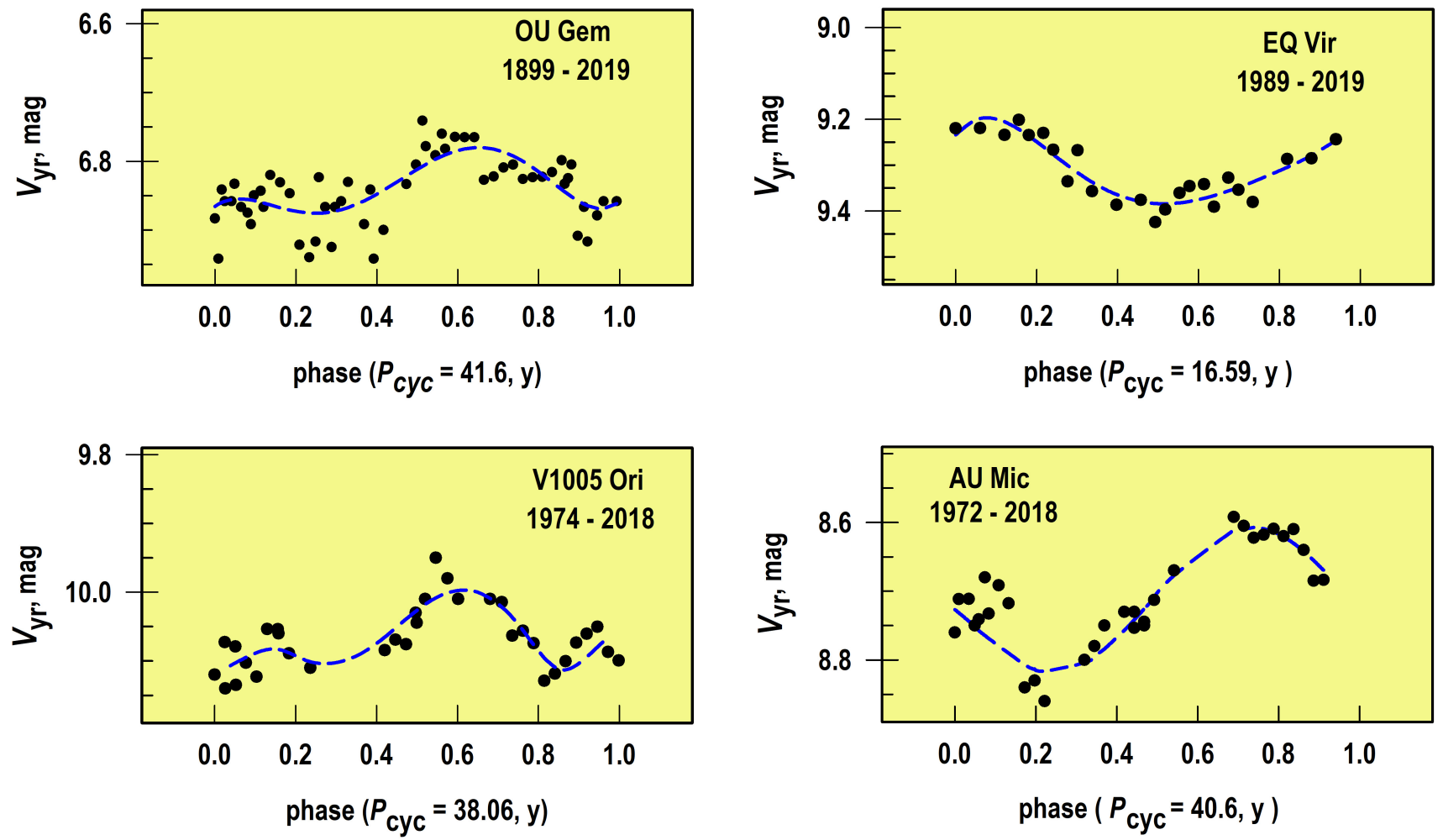

Figure 3. Long-term activity cycles in the studied stars. Filled circles present average annual $V$-values, dashed lines draw an approximation of phase curves by a high order polynomial and show the shape and amplitude of a possible cycle.

\section{ACTIVITY CYCLES AND MAGNETIC FIELDS}

The spots on star's surface are regions of an enhanced magnetic field, and therefore, the surface magnetic field of active stars is inhomogeneous. In this case, the average magnetic field is calculated as the sum of the weighted values of the magnetic field strength $\langle B\rangle=\Sigma\left|B_{i}\right| f_{i}$ in each of its elements with the coefficient $f_{i}$, the filling-factor, which expresses the area of the region covered by the magnetic field with the in- tensity $|B|_{i}$ in fractions of stellar surface area (Shulyak et al., 2014; 2017). In these works, the authors give a method for determining the average surface magnetic field on low-mass stars.

The G-M dwarfs studied by us were selected from the list of stars with measured magnetic fields (Shulyak et al., 2017). According to the data in the list, the average magnetic field is amplified in rapidly rotating fully convective $\mathrm{M}$ stars, for some of them $\langle B\rangle$ exceeds $4 \mathrm{kG}$. We tried to consider the effect of a surface magnetic field on the development of cyclic 
Table 2. Parameters of photospheric activity cycles and magnetic fields for the researched red dwarfs

\begin{tabular}{|c|c|c|c|c|c|c|c|}
\hline star name & spectral type & $B-V$ & $P_{c y c}, y$ & $A_{c y c}$, mag & ref & $\langle B\rangle, \mathrm{G}$ & ref \\
\hline LQ Hya & K2.0 & 0.91 & 18 & 0.27 & 6 & 2450 & 1 \\
\hline OU Gem & K2.0 & 0.95 & 41.6 & 0.09 & 9 & 2400 & 2 \\
\hline EQ Vir & K5.0 & 1.18 & 16.6 & 0.19 & 9 & 2000 & 3 \\
\hline V833 Tau & K5.0 & 1.04 & 78 & 0.6 & 7 & 1400 & 1 \\
\hline V1005Ori & M0.0 & 1.37 & 38.1 & 0.13 & 9 & 2600 & 4 \\
\hline AU Mic & M1.0 & 1.32 & 40.6 & 0.21 & 9 & 2300 & 5 \\
\hline DT Vir & M2.0 & 1.47 & 31.45 & 0.15 & 8 & 2600 & 4 \\
\hline AD Leo & M3.5 & 1.55 & 27 & 0.16 & 6 & 3100 & 4 \\
\hline DX Cnc & M6.5 & 2.05 & 2.67 & 0.10 & 8 & 3200 & 4 \\
\hline
\end{tabular}

References: (1) Saar S. H., 1996; (2) Saar \& Linsky, 1986; (3) Saar S. H. et al. 1986; (4) Shulyak et al., 2017; (5) Saar S. H., 1994; (6) Alekseev \& Kozhevnikova 2017; (7) Bondar' 2015; (8) Bondar' 2019; (9) this paper

photospheric activity according to photometric observations. As is known, the amplitude of the cycle is related to the area occupied by the spots: $A_{c y c}^{2} \sim S_{s p o t}$. To consider the relationship between cycle parameters and $\langle B\rangle$, we added in our sample $5 \mathrm{~K}-\mathrm{M}$ dwarfs, parameters of their cycles were taken from (Bondar', 2019). The list of stars, parameters of cycles and the mean magnetic fields are presented in Table 2.
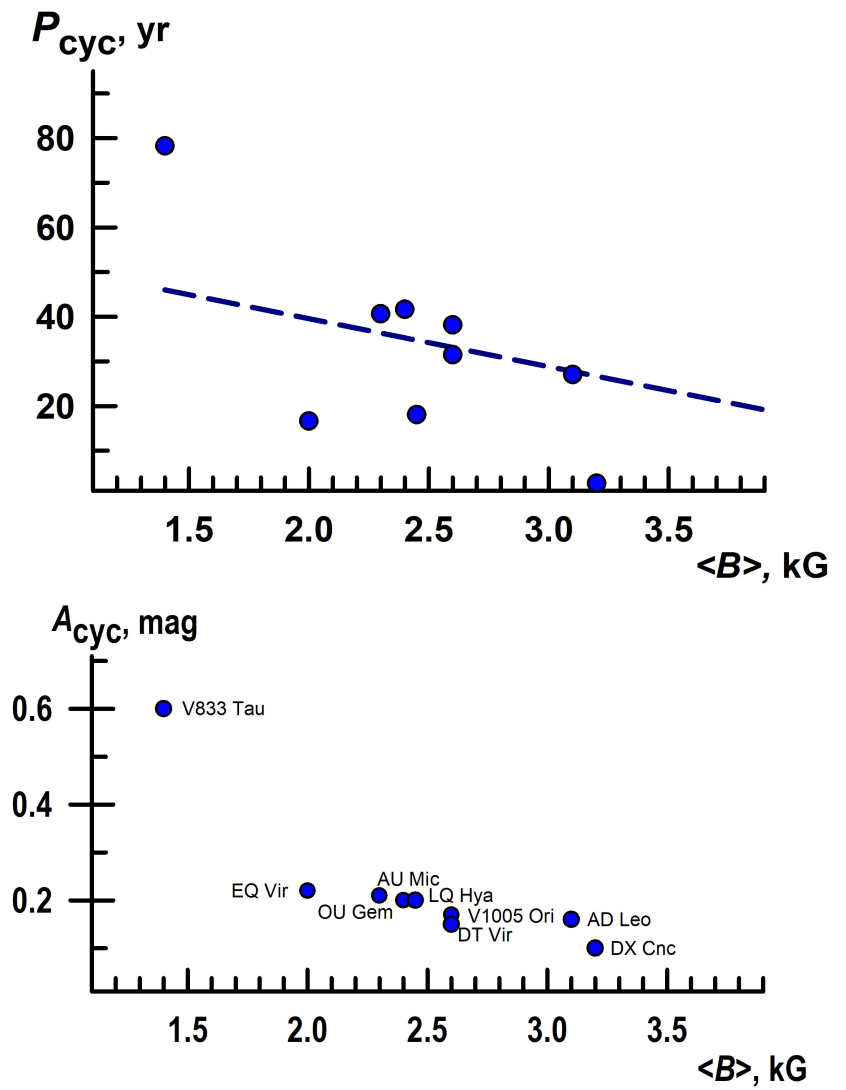

Figure 4. The relationship between the surface magnetic field and the activity cycle parameters of fast-rotating red dwarfs.
Besides V833 Tau, the averaged surface magnetic fields of the considered stars are 2-4 kG. For stars with such fields, the amplitude and duration of the cycle are increased with a decreasing of $\langle B\rangle$ that is displayed on Fig. 4. The limited number of the considered stars allows us only to propose the observed tendency. The deviations from the regression line that are noticeable in the graphs, as well as deviations of the cycle parameters for V833 Tau, can be caused both by errors in their determination and the actual existence of groups of stars with a specific type of relationship between the magnetic field and parameters of cycles. The question of whether it is possible to expect the development of long and high-amplitude cycles on stars with surface magnetic fields $<2 \mathrm{kG}$ requires further study.

\section{CONCLUSION}

The behavior of the average annual brightness of two Kdwarfs OU Gem and EQ Vir and the early M-dwarfs V1005 Ori and AU Mic was first studied on time span covers of several decades using photometry from databases for 1989-2019. Long-term series allowed us to determine maximum brightness values for the studied stars, all of them differ from those given in the astronomical SIMBAD database. The variability of the average annual brightness of all stars is cyclical.

The durations of possible cycles for OU Gem, V1005 Ori and AU Mic are 40-42 years, for EQ Vir - 16.6 years, and amplitudes are not more than $0.2^{m}$. Measurements of the magnetic fields of the stars studied by us showed that the average surface magnetic field strengths are several kilogauss. We examined the nature of the relationship between the parameters of the cycle, its duration and amplitude, and the value of $\langle B\rangle$, including another 5 stars with strong magnetic fields.

A noticeable tendency is an increase of the amplitude and duration of a cycle with a decrease in the value of $\langle B\rangle$. This may be due to the fact that with an increase in the surface magnetic field, it becomes more uniform and the level of 
activity changes to a lesser extent than in stars with strong local fields concentrated in large spots. It is known that the surface of M-dwarfs is covered with small but numerous spots.

\section{ACKNOWLEDGMENTS}

The authors are grateful to A.A. Shlyapnikov for help in working with databases, also to Z.A. Taloverova for technical help in preparation of text. In the work we used information from the SIMBAD database, photometric data from the Hipparcos, the International Variable Star Index (VSX) database, the Kamogata-Kiso-Kyoto Wide-Field Survey. The authors are thankful to all the staff providing the replenishment of these databases and access to them, as well as the developers of the AVE software package.

\section{FUNDING}

This work was carried out with the partial support of the Russian Foundation for Basic Research (grant 19-02- 00191a and 18-52-06002 Az_a).

\section{CONFLICT OF INTEREST}

The authors declare that they have no conflicts of interest.

\section{References}

[1] Alekseev I.Yu. Zapyatnennye zvezdy malych mass. Odessa: Astroprint, 137 pp. 2001. (rus.)

[2] Alekseev I.Yu., Kozhevnikova A.V. Long-term variations in the spottedness of BY Dra M dwarfs // Astron. Rep. V.61. No.3. P.221-232. 2017.

[3] Alekseev I.Yu., Bondar' N.I. Spottedness of the emissionline dwarf stars BF CVn, DT Vir, EQ Vir, and V1396 Cyg from photoelectric and photographic observations //Astron. Let. V.23. No.2. P.257-262. 1997.

[4] Argue A.N. UBV photometry of $300 \mathrm{G}$ and $\mathrm{K}$ type stars // MNRAS. V.125. P.557-570. 1963.

${ }^{[5]}$ Baliunas S.L., Nesme-Ribes E., Sokoloff D., Soon W.H. A dynamo interpretation of stellar activity cycles // Astrophys. J. V.460. P.848-854. 1996.

[6] Bondar' N.I. The long-term variability in active red dwarfs // Astron. Astrophys. Suppl. Ser. V.111. No.2. P.259-264. 1995.

[7] Bondar' N.I. The activity cycle of V833 Tau from photometric data for 1899-2009 // Astron. Rep. V.59. No.3. P.221-227. 2015.

[8] Bondar' N.I. Time-scales of activity cycles in solar-type stars and K-M dwarfs // Astron. Astrophys. Trans. V.31. No.3. P.295-302. 2019.

[9] Bopp B.W., Espenak F. Survey of the BY Draconis syndrome among dMe stars // Astron. J. V.82. P.916-924. 1977.
[10] Bopp B.W., Torres C.A., Busko I.C., Qust G.R. Observations of the BY Draconis Variable Gliese 182 // IBVS. No.1443. P.1. 1978.

[11] Byrne P.B., Doyle J.G., Butler C.J. Optical photometry and ultraviolet spectroscopy of the flare BY DRA star GL 182(V 1005 Ori) // MNRAS. V.206. P.907-918. 1984.

[12] Chabrier G., Baraffe I. Structure and evolution of lowmass stars // A\&A. V.327. P.1039-1053. 1997.

[13] Cutispoto G. Long-term monitoring of active stars. IV. UBV(RI)_c_ observations obtained at La Silla in December 1989 // Astron. Astrophys. Suppl. Ser. V.111. P.507. 1995.

[14] Cutispoto G. Long-term monitoring of active stars. VII. UBV(RI)_c photometry collected in March 1991 // Astron. Astrophys. Suppl. Ser. V.127. P.207-215. 1998.

[15] Cutispoto G., Messina S., Rodonò M. Long-term monitoring of active stars. IX. Photometry collected in $1993 / /$ A\&A. V.367. P.910-930. 2001.

[16] Cutispoto G., Messina S., Rodonò M. Long-term monitoring of active stars. X. Photometry collected in 1994 // A\&A. V.400. P.659-670. 2003.

[17] Distefano E., Lanza A. C., Lanza A. F., Messina S., Spada F. Activity cycles in members of young loose stellar associations // A\&A. V.606. A58. 14 pp. 2017.

[18] Ibañez Bustos R.V., Buccino A.P., Flores M., Martínez C. I., Maizel D., Messina S., Mauas P. J. D. First long-term activity study of AU Microscopii: a possible chromospheric cycle // MNRAS. V.483. P.1159-1167. 2019.

[19] Oláh K., Kővári Zs., Petrovay K., Soon W., Baliunas S., Kolláth Z., Vida K. Magnetic cycles at different ages of stars // A\&A. V. 590. id.A133. 13 pp. 2016.

[20] Phillips M.J., Hartmann L. Long-term variability of dMe stars // Astrophys. J. V.224. P.182-184. 1978.

[21] Pojmanski G. The all sky automated survey // Acta Astron. V.47. P.467-481. 1997.

[22] Reiners A., Basri G., Browning M. Evidence for Magnetic Flux Saturation in Rapidly Rotating M Stars // Astrophys. J. V.692. P.538-545. 2009.

[23] Saar S.H., Linsky J.L. New measurements of photospheric magnetic fields in late-type stars and emerging trends //Adv.Space Phys. V. 6. No.8. P.235-238. 1986.

[24] Saar S. H., Linsky J. L., Beckers J. M. The Magnetic Field of the BY Draconis Flare Star EQ Virginis // ApJ. V.302. P.777-784. 1986.

[25] Saar S. H. New Infrared Measurements of Magnetic Fields on Cool Stars // in: IAU Symposium V.154. Infrared Solar Physics / Eds. Rabin D. M., Jefferies J. T., Lindsey C. P.493-497. 1994.

[26] Saar S. H. Recent magnetic fields measurements of stellar magnetic fields // in: IAU Symposium V.176. Stellar 
Surface Structure / Eds. Strassmeier K. G., Linsky J. L. P. 237-244. 1996.

[27] Savanov I.S. Activity cycles of M dwarfs // Astron. Rep. V.56. P.716-721. 2012.

[28] Suárez Mascareño A., Rebolo R., González Hernández J.I. Magnetic cycles and rotation periods of late-type stars from photometric time series // A\&A. V.595. A12. 14 pp. 2016.

[29] Shulyak D., Reiners A., Seemann U., Kochukhov O., Piskunov N. Exploring the magnetic field complexity in $\mathrm{M}$ dwarfs at the boundary to full convection // A\&A. V.563. A35. 15 pp. 2014.

[30] Shulyak D., Reiners A., Engeln A., Malo L., Yadav R., Morin J., Kochukhov O. // Nature Astronomy. V.1. id.0184. 2017.

[31] Shulyak D., Reiners A., Nagel E., et al. Magnetic fields in M dwarfs from the CARMENES survey // A\&A. V.626. id.A86. 24 pp. 2019. 